\title{
Viscous spreading of non-Newtonian gravity currents in radial geometry
}

\author{
V. Di Federico ${ }^{1}$, S. Cintoli $^{1} \&$ G. Bizzarri ${ }^{2}$ \\ ${ }^{1}$ D.I.S.T.A.R.T. - Idraulica, Università di Bologna \\ ${ }^{2}$ Dipartimento di Architettura, Università di Ferrara
}

\begin{abstract}
A gravity current originated by a power-law viscous fluid propagating in axisymmetric geometry on a horizontal rigid plane below a fluid of lesser density is examined. The intruding fluid is considered to have a pure power-law constitutive equation. The set of equations governing the flow is presented, under the assumption of buoyancy-viscous balance and negligible inertial forces. The conditions under which the above assumptions are valid are examined and a selfsimilar solution in terms of a nonlinear ordinary differential equation is derived for the release of a fixed volume of fluid. The space-time development of the gravity current is discussed for different flow behavior indexes.

Keywords: non-Newtonian fluid, density current, gravity current, viscous flow, self-similar solution.
\end{abstract}

\section{Introduction}

Gravity currents, also termed density or buoyancy currents, are usually defined as flow of one fluid into another, driven by a density difference. These currents are mainly horizontal and are a common feature in many natural and artificial phenomena. Spreading of a gravity current along a rigid horizontal surface is governed by an interplay between buoyancy, inertial, and viscous forces. In the process, a gravity current passes through several distinct flow regimes which are characterized by the relative balance of forces. Immediately after its release, a gravity current usually experiences an adjustment phase that is strongly influenced by the release conditions. Subsequently, the balance between the buoyancy and inertial forces governs flow (this phase being thus termed the inertial regime) and holds until the current becomes so thin that viscous effects 
become comparable with the inertia of the current, if this ever happens. In this later stage (the viscous regime), flow is governed by the buoyancy and viscous forces. Typical currents which eventually evolve into the viscous regime include mudflows, lava flows, and those originating by discharge of effluents into rivers or lakes.

A large body of literature exists on gravity currents in different geophysical [1], environmental and industrial applications: for a review see Simpson [2, 3]. Horizontal gravity currents were studied by Hoult [4], Huppert and Simpson [5] and Didden and Maxworthy [6] among others. Huppert [7] derived, under a lubrication approximation, a spreading relationship (rate of advance of the front) for plane and axisymmetric gravity currents in the buoyancy-inertia and buoyancy-viscous regimes. His theoretical findings are in a good agreement with the experimental work by Huppert and Simpson [5], Didden and Maxworthy [6] and Maxworthy [8] for the release of a constant volume or constant inflow rate. Rottman and Simpson [9] extended these experimental results to the slumping phase of an inertial gravity current. There also exists a number of stability analyses of analytical solutions for inertial gravity currents $[10,11,12]$ and for viscous gravity currents [13]. Thomas et al. [14] and Marino and Thomas [15] included a porous substrate in their analysis of the inertial gravity currents. Ross et al. [16] incorporated in their analysis the effect of a sloping lower boundary.

Despite significant progress in understanding gravity currents of Newtonian fluids, there is a relatively poor number of studies of this phenomenon for nonNewtonian fluids. However, many fluids of geophysical or industrial interest exhibit a non-Newtonian rheology, with or without a yield stress. The simplest non-Newtonian rheological model is the Ostwald power-law model [17], which may be successful in describing the behavior of colloids, suspensions, fresh magma, and polymeric liquids. The power-law rheological model can also be seen as the asymptotic behavior of the Herschel-Bulkley model (in the limit as the yield stress tends to zero), which is widely adopted to describe flow of fine sediment-water mixtures $[18,19,20]$. Adopting a pure power-law model may facilitate the derivation of exact similarity solutions, such as that of Wilson and Burgess [21] for two-dimensional steady-state flow down a sloping plane. In the present paper, we derive the similarity solution for radial flow of a constant volume of a non-Newtonian power-law fluid with arbitrary flow behavior index. We do so in a way that generalizes earlier results of Huppert [7] obtained for a Newtonian fluid. Analogous results for the release of a fixed volume of fluid in plane geometry were obtained in [22] and later generalized for time dependent influx volume in [23].

\section{Flow modeling}

Consider a horizontal, radial gravity current of an incompressible nonNewtonian fluid of density $\rho$ at the bottom of an ambient fluid of depth $H$ and density $\rho-\Delta \rho$. (For the coordinate system see Fig. 1). In the shallow water approximation, the pressure distribution is hydrostatic, so that pressure satisfies (see [7]). 


$$
p=p_{0}+(\rho-\Delta \rho) g(H-h)+\rho g(h-z)
$$

where $\mathrm{h}$ is the non-Newtonian fluid depth and $p_{0}$ is the constant pressure at $z=$ $H$. Neglecting inertial forces, while accounting for buoyancy, gravitational, and viscous forces, results in the following momentum balance in cylindrical coordinates

$$
\frac{\partial p}{\partial r}+\frac{\partial \tau_{z r}}{\partial z}=0
$$

where $r, z$ are the radial and axial and coordinates, respectively; $p$ is pressure and $\tau_{z r}$ is shear stress. The validity of the simplified buoyancy-viscous balance (2) is explored in the Appendix, where the ratio between inertial and viscous forces is shown to be a decreasing function of time; thus, inertial forces are negligible for $t>t_{l}$, where $t_{l}$ is a threshold time value that renders equal inertial and viscous forces; its expression is derived explicitly in the Appendix.

An intruding fluid is considered to obey a pure power-law constitutive equation (see [17]).

$$
\tau_{z r}=-m\left|\frac{\partial u}{\partial z}\right|^{n-1} \frac{\partial u}{\partial z}
$$

where $u$ is radial velocity, $m$ consistency index, $n$ flow behavior index (a positive real number). When $n<1$, the model describes pseudoplastic (shear-thinning) behavior, whereas $n>1$ represents dilatant (shear-thickening) behavior. When $n$ equals unity, (3) reduces to the constitutive equation for a Newtonian fluid and $m$ becomes Newtonian viscosity $\mu$.

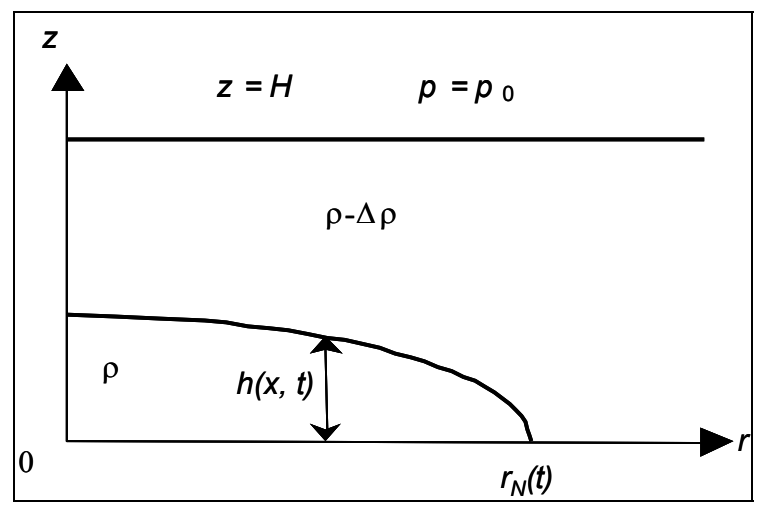

Figure 1: Sketch of flow domain.

Substituting (1) and (3) in (2) gives

$$
\rho g^{\prime} \frac{\partial h}{\partial r}-m n\left|\frac{\partial u}{\partial z}\right|^{n-1} \frac{\partial^{2} u}{\partial z^{2}}=0
$$


where $g^{\prime}=g \Delta \rho / \rho$ is reduced gravity. Eq. (4) is subject to the boundary conditions

$$
u(z=0)=0 ; \frac{\partial u}{\partial z}(z=h)=0
$$

The second condition in (5) implies that shear stress at the interface between the two fluids is much smaller than within the current. Validity of this assumption in the regime of a buoyancy-viscous balance can be demonstrated by following the argument of Huppert ([7], see his Appendix B). Integration of (4) with (5) yields the following expression for the velocity $u$

$$
u=-\frac{n}{(n+1) \frac{\rho g^{\prime}}{m} \frac{\partial h}{\partial r}}\left\{\left[\frac{\rho g^{\prime}}{m} \frac{\partial h}{\partial r}(z-h)\right]^{\frac{n+1}{n}}-\left[-\frac{\rho g^{\prime}}{m} h \frac{\partial h}{\partial r}\right]^{\frac{n+1}{n}}\right\}
$$

For one-dimensional transient flow, the mass conservation takes the form

$$
\frac{\partial h}{\partial t}+\frac{1}{r} \frac{\partial}{\partial r} \int_{0}^{h} r h(r, t) d z=0
$$

Substituting (6) into (7) yields

$$
\frac{\partial h}{\partial t}+\frac{n}{2 n+1} \frac{1}{r} \frac{\partial}{\partial r}\left[r h^{\frac{2 n+1}{n}}\left(-\frac{\rho g^{\prime}}{m} \frac{\partial h}{\partial x}\right)^{\frac{1}{n}}\right]=0
$$

Equation (8) defines the problem together with the global continuity equation requesting a fixed volume $Q$ to be released

$$
Q=2 \pi \int_{0}^{r_{N}(t)} r h(r, t) d r
$$

where $r N(t)$ is the radial coordinate of the head of the current.

\section{Solution to the problem and discussion}

Choosing $\bar{h}=Q^{1 / 3}$ as a typical length-scale, dimensionless (primed) variables are defined as $[22,23]$ :

$$
r=r^{\prime} \bar{h} ; \quad r_{\mathrm{N}}=r_{\mathrm{N}}^{\prime} \bar{h} ; h=h^{\prime} \bar{h} ; t=t^{\prime} /\left(\frac{\rho g^{\prime} \bar{h}}{m}\right)^{\frac{1}{n}}
$$


This recasts (8)-(9) in the dimensionless form (primes are dropped for convenience)

$$
\begin{gathered}
\frac{\partial h}{\partial t}+\frac{n}{2 n+1} \frac{1}{r} \frac{\partial}{\partial r}\left[r h^{\frac{2 n+1}{n}}\left(-\frac{\partial h}{\partial r}\right)^{\frac{1}{n}}\right]=0 \\
2 \pi \int_{0}^{r_{N}(t)} r h(r, t) d r=1
\end{gathered}
$$

By introducing the similarity variable

$$
\xi=\left(\frac{2 n+1}{n}\right)^{\frac{n}{3 n+5}} r t^{-\frac{n}{3 n+5}}
$$

and denoting the value of $\xi$ for $r=r N(t)$ by $\xi N$, the similarity solution of (11)(12) takes the form

$$
h(r, t)=\xi_{\mathrm{N}}^{(n+1) /(n+2)}\left(\frac{2 n+1}{n}\right)^{\frac{2 n}{3 n+5}} t^{-\frac{2 n}{3 n+5}} \Psi\left(\xi / \xi_{\mathrm{N}}\right)
$$

Substituting (13)-(14) in (11)-(12) yields respectively

$$
\frac{d}{d z}\left[z \Psi^{\frac{2 n+1}{n}}\left(-\frac{d \Psi}{d z}\right)^{\frac{1}{n}}\right]-\frac{n}{3 n+5} z^{2} \frac{d \Psi}{\partial z}-\frac{2 n}{3 n+5} z \Psi=0
$$

and

$$
\xi_{\mathrm{N}}=\left(2 \pi \int_{0}^{1} z \Psi d z\right)^{-\frac{n+2}{3 n+5}}
$$

where

$$
z=\frac{\xi}{\xi_{\mathrm{N}}}
$$

The solution to (15) is

$$
\Psi(z)=\left(\frac{n}{3 n+5}\right)^{n /(n+2)}\left(\frac{n+2}{n+1}\right)^{1 /(n+2)}\left(1-z^{n+1}\right)^{\frac{1}{n+2}}
$$


Substituting (18) into (16) gives

$$
\xi_{\mathrm{N}}=\left\{2 \pi \frac{1}{3 n+5}\left(\frac{n}{3 n+5}\right)^{n /(n+2)}\left(\frac{n+2}{n+1}\right)^{1 /(n+2)} \frac{\Gamma[2 /(n+1)] \Gamma[1 /(n+2)]}{\Gamma[(3 n+5) /((n+1)(n+2))]}\right\}^{-(n+2) /(3 n+5)}
$$

Finally, the (dimensionless) length of the gravity current is

$$
R=r_{\mathrm{N}}(t)=\xi_{\mathrm{N}}\left(\frac{n}{2 n+1}\right)^{\frac{n}{3 n+5}} t^{\frac{n}{3 n+5}}
$$

For $n=1$, governing equations and results reduce to those valid for a Newtonian fluid (see [1], [7]).

\section{Discussion and results}

Fig. 2 shows the shape of the function $\Psi(z)$ for $n=0.50,0.75,1.00,1.25,1.50$. The corresponding dynamics of the dimensionless current length is presented in Fig. 3. For $t<1$, the head of the current advances farther as $n$ decreases; the reverse is true for larger times.

Figs. 4, 5, and 6 illustrate how the gravity current develops in space and time, respectively for $n=0.50$ (pseudoplastic fluid), $n=1.00$ (Newtonian fluid), $n=$ 1.50 (dilatant fluid). In all cases, the rate of advance decreases (currents slow down) as time increases, as implied by (20). The prescribed fluid volume released slumps down more rapidly for dilatant gravity currents than for pseudoplastic ones: as a result, profiles of the former are more elongated than profiles of the latter.

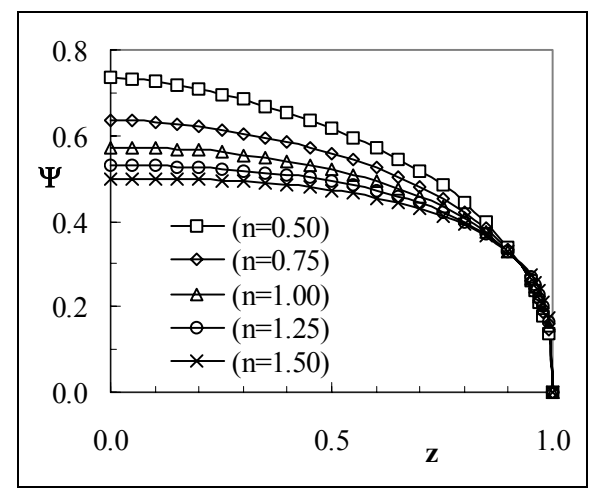

Figure 2: $\quad$ Shape of the current $\Psi(z)$ for various $n$. 


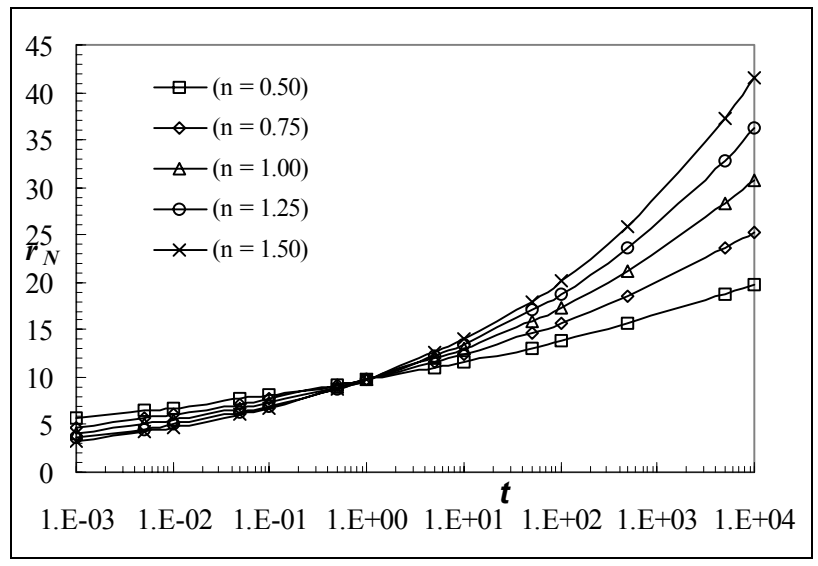

Figure 3: Dimensionless current length as a function of time for various $n$.

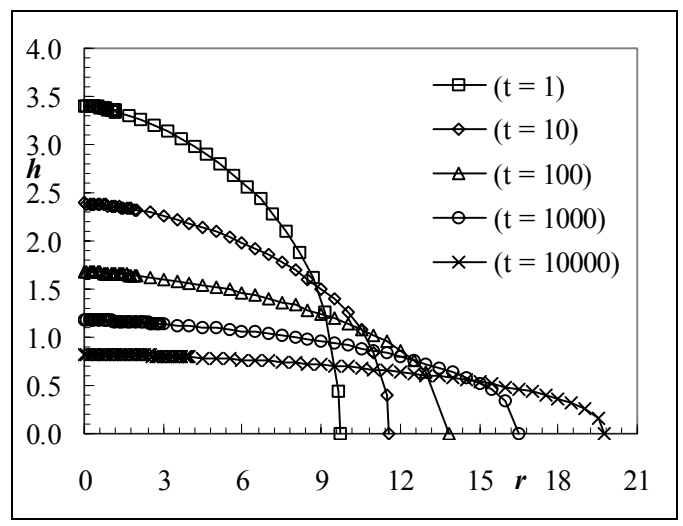

Figure 4: Profile of the current at different times for $n=0.50$.

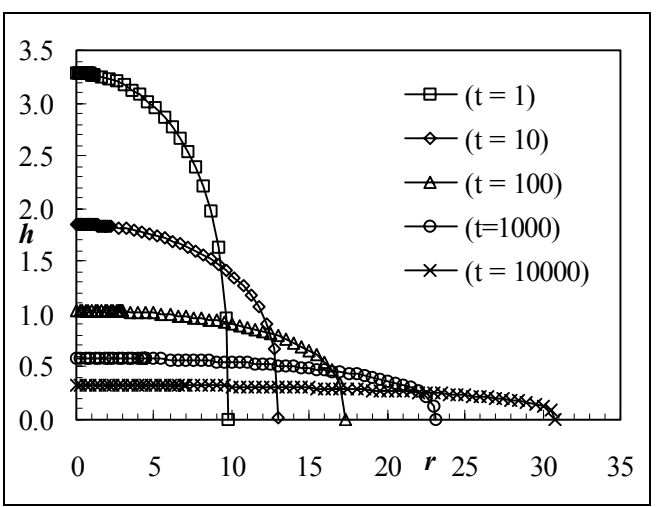

Figure 5: Profile of the current at different times for $n=1.00$. 


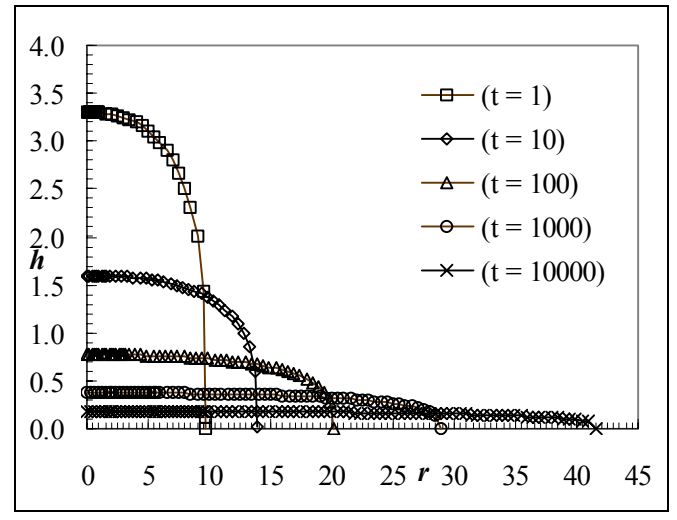

Figure 6: $\quad$ Profile of the current at different times for $n=1.50$.

\section{Summary and conclusions}

Our work leads to the following major conclusions: when studying horizontal gravity currents, at large times inertial forces are negligible as compared to buoyancy and viscous forces. Under the above assumption, we derive a set of equations which describe gravity currents of an incompressible power-law nonNewtonian fluid at the bottom of an ambient fluid of lower density propagating on a horizontal plane. The intruding fluid is considered to have a pure power-law constitutive equation. A self-similar solution is then derived for the release of a fixed volume of fluid, allowing one to study the development of the gravity current as a function of time and flow behavior index.

\section{Appendix range of validity of viscous regime}

The purpose of this Appendix is to determine the transition time $t_{l}$ when inertial and viscous forces are comparable. The order of magnitude of the fluid volume is $\approx h_{0} R^{2}$, where $h_{0}=Q / R^{2}$ is a representative thickness of the current and $R$ its radius. Buoyancy, $F_{g}$, inertial, $F_{i}$, and viscous, $F_{v}$, forces are given by

$$
\begin{gathered}
F_{g} \approx \rho g^{\prime} h_{0}^{2} R=\rho g^{\prime} Q^{2} R^{-3} \\
F_{i} \approx \rho U^{2} h_{0} R=\rho Q R t^{-2} \\
F_{v}=m\left(U / h_{0}\right)^{n} R^{2}=m Q^{-n} R^{2+3 n} t^{-n}
\end{gathered}
$$

where $U=R / t$ is a representative velocity of the current. For a current propagating in the inertial-buoyancy regime, equating (A1) and (A2) yields 


$$
R=\left(g^{\prime} Q\right)^{\frac{1}{4}} t^{\frac{1}{2}}
$$

as obtained by Huppert ([7], see Eq. (A5b)). Equating (A2) and (A3) under a viscous-buoyancy regime, and deriving $\mathrm{R}$ from the dimensional form of (20), yields

$$
\frac{F_{i}}{F_{v}}=\left(\frac{\rho^{4} Q^{n+3}}{m^{4} g^{\prime 3 n+1}}\right)^{\frac{1}{3 n+5}} t^{-2 \frac{n+5}{3 n+5}}
$$

Thus the (dimensional) transition time at which inertial and viscous forces are comparable is

$$
t_{1}=\left(\frac{\rho^{4} Q^{n+3}}{m^{4} g^{\prime 3 n+1}}\right)^{\frac{1}{2(n+5)}}
$$

Finally, it is worth noting that for $n=1$ all expressions in this Appendix reduce to the corresponding ones derived for a Newtonian fluid by Huppert ([7], see Appendix A).

\section{References}

[1] Huppert, H.E., The intrusion of fluid mechanics into geology, J. Fluid Mech., 173, pp. 557-594, 1986.

[2] Simpson, J.E., Gravity currents in the laboratory, atmosphere, and ocean, Ann. Rev. Fl. Mech., 14, pp. 213-234, 1982.

[3] Simpson, J.E., Gravity currents: in the environment and the laboratory, 2nd edition, Cambridge University Press, Cambridge, 1997.

[4] Hoult, D.P., Oil spreading on the sea, Ann. Rev. Fl. Mech., 4, pp. 341368, 1972.

[5] Huppert, H.E. and Simpson, J.E., The slumping of gravity currents, J. Fluid Mech., 99, pp. 785-799, 1980.

[6] Didden, N. and Maxworthy, T., The viscous spreading of plane and axysymmetric gravity currents, J. Fluid Mech., 121, pp. 27-42, 1982.

[7] Huppert, H.E., The propagation of two-dimensional and axisymmetric viscous gravity currents over a rigid horizontal surface, J. Fluid Mech., 121, pp. 43-58, 1982.

[8] Maxworthy, T., Gravity currents with variable inflow, J. Fluid Mech., 128, pp. 247-257, 1983.

[9] Rottman, J.W. and Simpson, J.E., Gravity currents produced by instantaneous release of a heavy fluid in a rectangular channel, J. Fluid Mech., 135, pp. 95-110, 1983.

[10] Grundy, R.E. and Rottman, J.W., The approach to self-similarity of the solution for the shallow water equations representing gravity currents releases, J. Fluid Mech., 156, pp. 39-53, 1985. 
[11] Grundy, R.E. and Rottman, J.W., Self-similar solutions of the shallow water equations representing gravity currents with variable inflow, J. Fluid Mech., 169, pp. 337-351, 1986.

[12] Gratton, J. and Vigo, C., Self-similar gravity currents with variable inflow revisited: plane currents, J. Fluid Mech., 258, pp. 77-104, 1994.

[13] Snyder, D. and Tait, S., A flow-front instability in viscous gravity currents, J. Fluid Mech., 369, pp. 1-21, 1998.

[14] Thomas, L.P., Marino, B.M. and Linden, P.F., Gravity currents over porous substrates, J. Fluid Mech., 366, pp. 239-258, 1998.

[15] Marino, B.M. and Thomas, L.P., Spreading of a gravity current over a permeable surface, J. of Hydr. Eng. ASCE, 128-5, pp. 527-533, 2002.

[16] Ross, A.N., Linden, P.F. and Dalziel, S.B., A study of three-dimensional gravity currents down a uniform slope, J. Fluid Mech., 453, pp. 239-261, 2002.

[17] Bird, R.B., Stewart, W.E. and Lightfoot, E.N., Transport Phenomena, Wiley, New York, 1960.

[18] Battaglia, M. and Borgia, A., Laminar flow of fine sediment water mixtures, J. Geophys. Res., 105-B3, pp. 5939-5945, 2000.

[19] Coussot, P., Proust, S. and Ancey, C., Rhelogical interpretation of deposits of yield stress fluids, J. Non-Newtonian Fluid Mech., 66, pp. 55$70,1996$.

[20] Di Federico, V., Permanent waves in slow free-surface flow of a Herschel-Bulkley fluid, Meccanica, 33-2, pp. 127-137, 1998.

[21] Wilson, S.D.R. and Burgess, S.L., The steady, spreading flow of a rivulet of mud, J. Non-Newtonian Fluid Mech., 79, pp. 77-85, 1998.

[22] Di Federico, V. and Guadagnini, A., Propagation of a plane nonNewtonian gravity current, Proc. of the 28th IAHR Congress, Graz, Austria 1999, Abstract Volume (Papers on CD-ROM), p. 284, 1999.

[23] Di Federico, V., Cintoli, S. and Malavasi, S., Viscous spreading of nonNewtonian gravity currents on a plane, Meccanica, in press, 2006. 